\title{
A NEW PROCEDURE FOR STOCHASTIC REALIZATION OF SPECTRAL DENSITY MATRICES*
}

\author{
A. J. VAN DER SCHAFT $\dagger \ddagger$ AND J. C. WILLEMS $\dagger$
}

\begin{abstract}
In this paper we consider the problem of obtaining a state space realization of a zero mean gaussian vector process. A new algorithm is presented for the case in which the process is given in terms of its spectral density function. Contrary to the usual procedure followed, which requires a partial fraction expansion, the algorithm presented starts with a (deterministic) realization of the spectral density function itself.
\end{abstract} equation

Key words. Spectral densities, stochastic realization, Hamiltonian systems, spectral factorization, Riccati

1. Introduction. One of the basic problems in mathematical system theory is the question of obtaining an "internal" state space realization of a system given in "external" form. In the stochastic case the main problem studied in this context may be formulated as follows: Given a stationary zero mean gaussian vector process y, construct a stationary zero mean Gauss-Markov vector process $x$ and a matrix $C$ such that $C x$ has the same statistical properties as $y$.

The basic formulation of this problem is due to Kalman [6] about fifteen years ago and since that time numerous publications have appeared on it. A good account of the existing theory may be found in the book by Faurre et al. [5]. The existing algorithms assume that the process $y$ is given by its (matrix) autocorrelation function. However, in many applications the process $y$ will be given by its spectral density function and in order to apply the existing algorithms it is then necessary to factor (e.g., by means of a partial fraction expansion) the spectral density into a part which is analytic in $\operatorname{Re} s>0$ and a part which is analytic in $\operatorname{Re} s<0$. This is however a nontrivial step in the application of these methods, and more often than not this difficulty is glossed over. Indeed this requires the factorization of a high order polynomial. From a numerical point of view this is a nonlinear problem of the same level of difficulty as solving an algebraic Riccati equation. This difficulty motivates the approach followed in the present paper. We will propose a different solution of the problem of stochastically realizing a spectral density. Our approach is based on the observation that a spectral density matrix $\Phi(s)$ is always Hamiltonian $\left(\Phi(s)=\Phi^{T}(-s)\right)$ and passive $\left(\Phi(j \omega)+\Phi^{T}(-j \omega) \geqq 0\right)$. Exploiting this structure, one may obtain a special "deterministic" realization of $\Phi(s)$ (viewed itself as a rational function in $s$ ) which then yields an algebraic Riccati equation from which a stochastic realization (or a white noise representation) of the original process is readily derived.

2. Realization of autocorrelation functions. In this section we will introduce the problem and review some of the main results previously obtained on it.

Let $y:=\left\{y_{t}, t \in \mathbb{R}\right\}$ be a real $p$-dimensional vector process defined on a probability space $\{\Omega, \mathscr{A}, P\}$. We assume that $y$ is gaussian, zero mean and stationary. In the stochastic realization problem for such processes we look for a real $n$-dimensional zero mean stationary Gauss-Markov process $x:=\left\{x_{t}, t \in \mathbb{R}\right\}$, defined on a probability

\footnotetext{
* Received by the editors September 12, 1982, and in revised form September 21, 1983.

$\dagger$ Mathematics Institute, P.O. Box 800, 9700 AV Groningen, the Netherlands.

$\ddagger$ Current address: Department of Applied Mathematics, Twente University of Technology, P.O. Box 217, 7500 AE Enschede, the Netherlands.
} 
space $\left\{\Omega^{\prime}, \mathscr{A}^{\prime}, P^{\prime}\right\}$, and a matrix $C: \mathbb{R}^{n} \rightarrow \mathbb{R}^{p}$ such that $C x \sim y$. Here $\sim$ denotes (stochastic) equivalence, i.e. the probability distributions of the vectors $\left(y_{t_{1}}, y_{t_{2}}, \cdots, y_{t_{k}}\right)$ and $\left(C x_{t_{1}}, C x_{t_{2}}, \cdots, C x_{t_{k}}\right)$ should be equal for all choices of $t_{1}, t_{2}, \cdots, t_{k}$. Obviously if $(x, C)$ realizes $y$, then so does $\left(P x, C P^{-1}\right)$ for any nonsingular matrix $P$. We will say that $(x, C)$ defines a minimal realization of $y$ if the only matrices $P: \mathbb{R}^{n} \rightarrow \mathbb{R}^{n}$ such that $\left(P x, C^{\prime}\right)$ also realizes $y$ for some $C^{\prime}$ are actually the nonsingular matrices.

Note that we do not require that $y$ and $x$ are defined on the same probability space. Often this problem is therefore called the weak realization problem in order to distinguish it from the strong version in which $\left\{\Omega^{\prime}, \mathscr{A}^{\prime}, P^{\prime}\right\}=\{\Omega, \mathscr{A}, P\}$. Other than in Remark 6 we will in this paper deal exclusively with the weak version.

The marginal probability distributions of $y$ (and thus of all processes which are equivalent to it) are completely specified by its autocorrelation function $R: \mathbb{R} \rightarrow \mathbb{R}^{p \times p}$ defined by $R(t):=\mathbf{E}\left\{y(t) y^{T}(0)\right\}$. The restriction of $R$ to $[0, \infty)$ will be denoted by $R^{+}$. Of course, $R^{+}$specifies $R$ completely since $R(t)=R^{T}(-t)$. The following basic result from deterministic realization theory is well known:

Proposition 1. The following conditions are equivalent:

(i) $R^{+}$is Bohl (i.e., every entry of $R^{+}$is a finite sum of products of a polynomial, an exponential, and a trigonometric function).

(ii) There exists matrices $(F, G, H)$ such that $R^{+}(t)=H e^{F t} G$, with $F \in \mathbb{R}^{n \times n}, G \in$ $\mathbb{R}^{n \times p}$, and $H \in \mathbb{R}^{p \times n}$.

In addition:

(iii) There is a minimal $n, n_{\min }$, for which the factorization in (ii) is possible. This $n_{\min }$ is called the McMillan degree of $R^{+}$, and $n=n_{\min }$ iff $(F, G)$ is controllable and $(F, H)$ is observable. The triple $(F, G, H)$ is then called minimal.

(iv) All triples $(F, G, H)$ with $n=n_{\min }$ are obtainable from one by the transformation group

$$
(F, G, H) \underset{\operatorname{det} S \neq 0}{\stackrel{s}{\longrightarrow}}\left(S F S^{-1}, S G, H S^{-1}\right)
$$

Assume that $x$ is an $n$-dimensional zero mean stationary Gauss-Markov process with $E\left\{x(t) x^{T}(t)\right\}=Q>0$ (obviously $Q>0$ whenever $(x, C)$ is a minimal realization of $y$ ). We call such Markov processes nonsingular. Then $\mathbf{E}\{x(t) \mid x(0)\}$ for $t \geqq 0$ is of the form $e^{A t} x(0)$ for some $A$. Moreover it is easily seen that $Q$ and $A$ completely specify the marginal distributions of the Markov process $x$. It is in fact not difficult to see that this way any pair of matrices $\{Q, A\}$ will define a zero mean stationary nonsingular Gauss-Markov process $x$ with $\mathbf{E}\left\{x(0) x^{T}(0)\right\}>0$ iff $Q=Q^{T}>0$ and $A Q+$ $Q A^{T} \leqq 0$. We will call $Q$ the covariance and $A$ the infinitesimal generator of $x$. Now the marginal distributions of the process $C x$ are completely specified by $(A, Q, C)$ and it thus makes sense to talk about this triple as defining a realization. Since in our context minimality as defined previously corresponds also to taking the dimension of $x$ as small as possible we will call a realization $(x, C)$ with $x n$-dimensional and $n$ as small as possible a minimal realization.

Two processes $x_{1}$ and $x_{2}$ will be called linearly equivalent if there exists a nonsingular matrix $S$ such that $x_{2} \sim S x_{1}$. Following this we will call two realizations $\left(A_{1}, Q_{1}, C_{1}\right)$ and $\left(A_{2}, Q_{2}, C_{2}\right)$ linearly equivalent if there exists a nonsingular matrix $S$ such that $\left(A_{2}, Q_{2}, C_{2}\right)=\left(S A_{1} S^{-1}, S Q_{1} S^{T}, C_{1} S^{-1}\right)$. The following theorem is the basic result in this area. It follows from the basic work by Kalman [6] and Faurre [4] and has later been studied further by Anderson [2], Lindquist and Picci [8], Ruckebush [11] and many others. 


\section{THEOREM 2.}

(i) There exists a finite dimensional realization of y iff its autocorrelation function $R$ is Bohl.

(ii) All minimal realizations $(A, Q, C)$ can, up to linear equivalence, be obtained from a minimal factorization triple $(F, G, H)$ of $R^{+}$by taking $A=F, C=H$, and solving the relations

$$
F G+Q F^{T} \leqq 0, \quad Q H^{T}=G
$$

for $Q=Q^{T}$

The problem thus becomes one of solving this combination of matrix inequalities/equalities. Actually, it may be shown [4], [5], [13] that there exist solutions $Q_{-}, Q_{+}$such that for every other solution $Q$, there holds $0<Q_{-} \leqq Q \leqq Q_{+}<\infty$. Moreover the solution set is convex and compact. A great deal of additional information on the structure of the solution set of these equations may be found in the above references. Note that choosing $A=F$ and $C=H$ in the above theorem corresponds to fixing the basis in the state space (since $(F, H)$ is observable). Once the basis has been picked, it is only the covariance of $x, Q$, which remains to be chosen.

The above results are the well-known basic facts of stochastic realization theory. They yield a minimal stochastic realization by the following procedure. This procedure inputs as the

Data. The autocorrelation $R^{+}$of $y$.

Then it computes as

Step 1. Determine a minimal realization $(F, G, H)$ of $R^{+}$.

Subsequently it proceeds with

Step 2. Solve the linear matrix inequality

$$
Q=Q^{T}, \quad F Q+Q F^{T} \leqq 0, \quad Q H^{T}=G .
$$

The procedure then returns $(F, Q, H)$ which defines a minimal realization $(x, H)$ of $y$, where $x$ is a Gauss-Markov process with convariance $Q$ and infinitesimal generator $F$. For the algorithmic implementation of Step 1 we can use any of the realization theory algorithms of linear systems theory. Also Step 2 has received much attention and may be reduced to solving linear matrix equations and a suitably defined reduced order algebraic Riccati equation.

Remark 1. Some readers may be more familiar with the problem of generating a spectral density function by passing white noise through a linear system (which is called the shaping filter). In fact, this problem is solved by a simple extension of the result of Theorem 2 . Indeed, let $(A, Q, C)$ be a minimal stochastic realization and let $B$ be such that $A Q+Q A^{T}=-B B^{T}$. Consider now the system described by the stochastic differential equation

$$
\begin{aligned}
& d x=A x d t+B d w, \quad x(0)=x_{0}, \\
& \tilde{y}=C x
\end{aligned}
$$

with $x_{0}$ zero mean gaussian, and $\mathbf{E}\left\{x_{0} x_{0}^{T}\right\}=Q$, and with $w:=\left\{w_{t}, t \in[0, \infty)\right\}$ a normalized Wiener process, independent of $x_{0}$. This defines a white noise driven model which generates a process $\tilde{y} \sim y$.

In the present paper we will develop an algorithm which starts from $\Phi$ and proceeds by determining a minimal realization of $\Phi$ (considered as a rational function in $s$ ). Since in very many applications the spectral density is a more basic design specification than the autocorrelation function, it may be of interest to have this alternative algorithm 
available (even though we do not claim any superiority of our algorithm above the previously mentioned one which uses the partial fraction expansion followed by an implementation of Theorem 2).

3. Realization of spectral density matrices. Let $y=\left\{y_{t}, t \in \mathbb{R}\right\}$ be a real gaussian $p$-dimensional vector process and assume that its autocorrelation function $R$ is integrable. Let $\Phi(s)$ denote the (two-sided) Laplace transform of $R$. $\Phi$ is called the spectral density of $y$ [10], [16]. Obviously $\Phi$ is well-defined in a strip containing the imaginary axis. The existence of a finite dimensional realization of $y$ may of course also be deduced from its spectral density $\Phi$ :

Proposition 3. Assume that $R$ is integrable. Then the following conditions are equivalent:

(i) $R^{+}$is Bohl.

(ii) $\Phi$ is rational.

We remark that the assumption that $R$ is integrable is in our context equivalent to assuming that the process $y$ is ergodic.

In applications of stochastic realization theory it happens more often than not that one starts with a rational spectral density matrix. Of course, in this case one may proceed by computing a partial fraction expansion of $\Phi(s)$

$$
\Phi(s)=Z(s)+Z^{T}(-s)
$$

with $Z(s)$ analytic in $\operatorname{Re} s \geqq 0$, realizing $Z(s)$ minimally as $Z(s)=H(s I-F)^{-1} G$, and using the theory of $\S 2$. However, the problem of factoring $\Phi(s)$ into the above form is a highly nontrivial one. It requires factoring a polynomial which may be of high degree, and all together this may very well be the most difficult step in this whole realization procedure.

Our purpose in the present paper is to outline a procedure which proceeds by using a realization of the spectral density $\Phi(s)$ directly (and not of its "causal" part $Z(s))$. It is well known that a rational $(p \times p)$ matrix $\Phi(s)$ is a spectral density matrix of a process $y$ with integrable $R$ if and only if it has the following properties:

(i) $\Phi(s)=\Phi^{T}(-s)$,

(ii) $\Phi(s)$ has no poles on the imaginary axis

(iii) $\Phi(j \omega) \geqq 0 \quad \forall \omega \in \mathbb{R}$,

(iv) $\lim _{s \rightarrow \infty} \Phi(s)=0$.

In the sequel we will show how by constructing a special minimal realization of $\Phi(s)$, initially seen as a transfer function, we can solve the stochastic realization problem. The key observation for our procedure is that $\Phi(s)$ is a Hamiltonian transfer matrix, i.e. $\Phi(s)=\Phi^{T}(-s)$ (in the literature this property is also called para-hermitian). It is well known (cf. [3]) that a minimal realization $(\bar{A}, \bar{B}, \bar{C})$ of $\Phi(s)$ will then satisfy

$$
\begin{aligned}
& \bar{A}^{T} J+J \bar{A}=0, \\
& \bar{B}^{T} J=\bar{C}
\end{aligned}
$$

for some unique nonsingular matrix $J$ satisfying $J=-J^{T}$. It follows from the nonsingularity of $J$ that the dimension of the state space is even, say $2 n$. Basis free, $J$ is an anti-symmetric bilinear form on $\mathbb{R}^{2 n}$, and is called a symplectic form. By Darboux's theorem there exist bases of $\mathbb{R}^{2 n}$ in which $J$ has the matrix form $\left(\begin{array}{cc}0 & -I_{n} \\ I_{n} & 0\end{array}\right)$ (cf. [1]).

The second observation which we make is that $\Phi(j \omega) \geqq 0$ implies the passivity of the system with transfer matrix $\Phi$. (Note that $\Phi$ is not asymptotically stable. Passivity here means that for any input/output pair which corresponds to a closed path in state space, the $\mathscr{L}_{2}$ inner product of input and output is nonnegative (see (14])). In fact 
$\Phi(j \omega) \geqq 0$ is equivalent to the existence of a nonsingular symmetric matrix $\Sigma$, which is not necessarily positive definite (basis free, $\Sigma$ is a nondegenerate quadratic form), such that (see [13])

$$
\begin{aligned}
& \bar{A}^{T} \Sigma+\Sigma \bar{A} \leqq 0, \\
& \bar{B}^{T} \Sigma=\bar{C} .
\end{aligned}
$$

We emphasize that the solution $\Sigma$ of (3) and (4) is in general not unique. The facts that the realizations satisfy the symmetry conditions (1), (2) and the passivity conditions (3), (4) may be combined to yield:

LEMMA 4. There exists a solution $\Sigma$ of $(3,4)$ such that

$$
\Sigma=J \Sigma^{-1} J
$$

Proof. Notice that if $\Sigma$ satisfies (3) and (4), then also $J \Sigma^{-1} J$ does, because of (1) and (2). Furthermore it is well known that the set of all $\Sigma$ 's satisfying (3) and (4) is convex and compact. Since the map $\Sigma \rightarrow J \Sigma^{-1} J$, considered as a map on the space of nonsingular symmetric matrices, is continuous it therefore follows from Brouwer's fixed point theorem that there exist $\Sigma$ 's satisfying (3) and (4) and $\Sigma=J \Sigma^{-1} J$.

Remark 2. The proof of Lemma 4 is completely analogous to the existence of reciprocal passive realizations [15].

Now consider for a solution $\Sigma$ of (3), (4) which satisfies (5), the matrix $J^{-1} \Sigma$ (this is the Hamiltonian matrix corresponding to the energy function $\left.\frac{1}{2} x^{T} \Sigma x\right)$. Because of (5) and $\Sigma=\Sigma^{T}, J=-J^{T}$ it follows that

$$
\begin{aligned}
& \left(J^{-1} \Sigma\right)^{T} J\left(J^{-1} \Sigma\right)=-J \\
& \left(J^{-1} \Sigma\right)^{2}=I .
\end{aligned}
$$

In [9] (see also [12]) it is proven that therefore there exist bases of $\mathbb{R}^{2 n}$ in which

$$
J=\left(\begin{array}{cc}
0 & -I_{n} \\
I_{n} & 0
\end{array}\right) \text { and } J^{-1} \Sigma=\left(\begin{array}{cc}
I_{n} & 0 \\
0 & -I_{n}
\end{array}\right)
$$

or equivalently

$$
J=\left(\begin{array}{cc}
0 & -I_{n} \\
I_{n} & 0
\end{array}\right) \text { and } \Sigma=\left(\begin{array}{cc}
0 & I_{n} \\
I_{n} & 0
\end{array}\right)
$$

Roughly, this may be seen as follows. From (6) and (7) it follows that the eigenvalues of $J^{-1} \Sigma$ are +1 (multiplicity $n$ ) and -1 (multiplicity $n$ ). Define $\Sigma^{+}:=\operatorname{ker}\left(I-J^{-1} \Sigma\right.$ ) and $\Sigma^{-}:=\operatorname{ker}\left(I+J^{-1} \Sigma\right)$. Take an arbitrary basis $q_{1}, \cdots, q_{n}$ of $\Sigma^{+}$. It can be proven that there exists a basis $p_{1}, \cdots, p_{n}$ of $\Sigma^{-}$such that $q_{i}^{T} J p_{j}=\delta_{i j}, i, j=1, \cdots, n$. Furthermore it can be seen that after having fixed $q_{1}, \cdots, q_{n}$, the vectors $p_{i}$ as above are uniquely determined. Then in such a basis $\left\{q_{1}, \cdots, q_{n}, p_{1}, \cdots, p_{n}\right\}, J$ and $\Sigma$ have the required form (8). (Notice that the transformations which leave $J$ and $\Sigma$ in the form (8) are exactly the transformations of the form

$$
\left(\begin{array}{cc}
S & 0 \\
0 & \left(S^{T}\right)^{-1}
\end{array}\right),
$$

with $\operatorname{det} S \neq 0$.) 
Proceeding with a basis as explained it follows from (1) and (2) that $\bar{A}, \bar{B}$ and $\bar{C}$ have the form

$$
\bar{A}=\left(\begin{array}{cc}
F & -P \\
-R & -F^{T}
\end{array}\right), \quad \bar{B}=\left(\begin{array}{c}
G \\
H^{T}
\end{array}\right), \quad \bar{C}=\left(\begin{array}{ll}
H & -G^{T}
\end{array}\right)
$$

with $P=P^{T}$ and $R=R^{T}$.

Then (3) is equivalent to $P \geqq 0$ and $R \geqq 0$, while (4) gives $G=0$.

We sum this up in

THEOREM 5. Let $\Phi(s)$ be a matrix of rational functions satisfying

(i) $\Phi(s)=\Phi^{T}(-s)$,

(ii) $\Phi$ has no poles on the imaginary axis,

(iii) $\Phi(\dot{j \omega}) \geqq 0 \forall \omega \in \mathbb{R}$,

(iv) $\lim _{s \rightarrow \infty} \Phi(s)=0$.

Then there exists a minimal realization $(\bar{A}, \bar{B}, \bar{C})$ of $\Phi(s)$ such that

$$
\begin{aligned}
& \bar{A}=\left(\begin{array}{cc}
F & -P \\
-R & -F^{T}
\end{array}\right), \quad P=P^{T} \geqq 0, \quad R=R^{T} \geqq 0, \\
& \bar{B}=\left(\begin{array}{c}
0 \\
H^{T}
\end{array}\right), \quad \bar{C}=\left(\begin{array}{ll}
H & 0
\end{array}\right) .
\end{aligned}
$$

The next step is to consider the following ( $n$-dimensional) Riccati equation

$$
F^{T} K+K F-K P K+R=0 .
$$

We first state

Lemma 6. Controllability of $(\bar{A}, \bar{B})$ implies controllability of $(F, P)$.

Proof. Write $x=\left(\begin{array}{l}x_{1} \\ x_{2}\end{array}\right)$ corresponding to $\left(\begin{array}{cc}F & -P^{T} \\ -R & -F\end{array}\right)$, with $x_{1} \in X_{1}, x_{2} \in X_{2}$ (with $X_{1} \simeq$ $\left.X_{2} \simeq \mathbb{R}^{n}\right)$. Suppose that $(F, P)$ is not controllable. Then there exists a subspace $\mathscr{L} \subset X_{1}$, and $\mathscr{L} \neq X_{1}$, such that $\operatorname{Im} P \subset \mathscr{L}$ and $\mathscr{L}$ is $F$-invariant. Then it can be easily checked that $\mathscr{L} \oplus X_{2}$ is invariant with respect to $\left(\begin{array}{cc}F & -P_{T} \\ -R & -F^{T}\end{array}\right)$ and contains $\operatorname{Im}\left({ }_{H^{T}}{ }^{T}\right)$. Therefore it follows that

$$
\left(\begin{array}{cc}
F & -P \\
-R & -F^{T}
\end{array}\right),\left(\begin{array}{c}
0 \\
H^{T}
\end{array}\right)
$$

is not controllable. $(F, R)$.

Remark 3. In general, observability of $(\bar{A}, \bar{C})$ does not imply observability of

Consider now the Riccati equation (11). By Lemma $6 P=P^{T} \geqq 0$ is such that $(F, P)$ is controllable. Since also $R=R^{T} \geqq 0$, and $\bar{A}$ does not have purely imaginary eigenvalues, this implies [7], [13] that there exists a symmetric nonnegative definite matrix $K$ satisfying (11) and such that $F-P K$ is asymptotically stable. In fact, we have to take the maximal $K=K^{T}$ satisfying (11) [13].

Applying now the symplectic transformation $\bar{K}=\left(\begin{array}{cc}I & 0 \\ -K & I\end{array}\right)$ to $\bar{A}, \bar{B}$ and $\bar{C}$ yields

$$
\bar{K} \bar{A} \bar{K}^{-1}=\left(\begin{array}{cc}
F-P K & -P \\
0 & -(F-P K)^{T}
\end{array}\right), \quad \bar{K} \bar{B}=\left(\begin{array}{c}
0 \\
H^{T}
\end{array}\right), \quad \bar{C} \bar{K}^{-1}=\left(\begin{array}{ll}
H & 0
\end{array}\right) .
$$

Now define $A:=F-P K, C:=H$, and let $Q$ be the symmetric positive definite matrix satisfying

$$
A Q+Q A^{T}=-P
$$

( $Q$ is uniquely determined by (13) since $\operatorname{Re} \sigma(A)<0$ and $Q$ is positive definite since 
$(A, P)$ is controllable.) Then $(A, Q, C)$ is a minimal realization of the process with spectral density $\Phi(s)$. We summarize this as

THEOREM 7. Let $\Phi(s)$ be a rational spectral density matrix. Then $\Phi(s)$ has, as a transfer matrix, a minimal realization $(\bar{A}, \bar{B}, \bar{C})$ of the form

$$
\bar{A}=\left(\begin{array}{cc}
F-P K & -P \\
0 & -(F-P K)^{T}
\end{array}\right), \quad \bar{B}=\left(\begin{array}{c}
0 \\
H^{T}
\end{array}\right), \quad \bar{C}=\left(\begin{array}{ll}
H & 0
\end{array}\right)
$$

with $P=P^{T} \geqq 0$, and $F-P K$ asymptotically stable. Now let $A:=F-P K, C:=H$ and let $Q=Q^{T}>0$ be the unique solution of $A Q+Q A^{T}=-P$. Then $(A, Q, C)$ is a minimal realization of the process with spectral density $\Phi(s)$.

Proof. The existence of a minimal realization $(A, B, C)$ as above follows from the previous considerations (Theorem 5 and Lemma 6). Then it is easy to see that

$$
\bar{C}(I s-\bar{A})^{-1} \bar{B}=H(I s-A)^{-1} P\left(-I s-A^{T}\right)^{-1} H^{T} .
$$

Write $P=B B^{T}$ and define $W(s)=H(I s-A)^{-1} B$; then it follows that $\Phi(s)=$ $W(s) W^{T}(-s)$. Furthermore $W(s)$ is analytic in $\operatorname{Re} s \geqq 0$ and $(A, B, H)$ is a minimal triple. So we have given a spectral factorization of $\Phi(s)$, which is known to be equivalent to the stochastic realization problem (see for instance [2], [5]). It is well known that in terms of this factorization the covariance $Q$ of the associated Markov process is given as the solution of $A Q+Q A^{T}=-B B^{T}=-P$.

Remark 4. Hence we have constructed a spectral factorization $\Phi(s)=$ $W(s) W^{T}(-s)$ directly from $\Phi(s)$, instead of first taking a partial fraction expansion $\Phi(s)=Z(s)+Z^{T}(-s)$.

Remark 5. Write $P=B B^{T}$; then Theorem 7 gives us immediately a white noise representation of the process

$$
\begin{aligned}
& d x=A x d t+B d w, \quad x(0)=x_{0}, \\
& \tilde{y}=C x
\end{aligned}
$$

with $x_{0}$ zero mean gaussian, $\mathbf{E}\left\{x_{0} x_{0}^{T}\right\}=Q$ and $w:=\left\{w_{t}, t \in[0, \infty)\right\} a$ normalized Wiener process independent of $x_{0}$ (see Remark 1 ). In this case, since $A$ is asymptotically stable, we can equivalently define $x_{t}$ by

$$
x_{t}=\int_{-\infty}^{t} e^{A(t-s)} B d w(s) .
$$

Remark 6. It is well known (cf [4]) that a strong realization corresponds to a $P$ of minimal rank. In our construction we obtain a strong realization by taking $\Sigma=\Sigma^{T}$ satisfying (3), (4) and (5) such that $\bar{A}^{T} \Sigma+\Sigma \bar{A}$ restricted to $\Sigma^{+}$has minimal rank.

Remark 7. Take $Q=Q^{T}>0$ as the solution of $A Q+Q A^{T}=-P$. Applying the (symplectic) transformation $\bar{Q}=\left(\begin{array}{cc}I & Q \\ 0 & I\end{array}\right)$ to

$$
\bar{A}=\left(\begin{array}{cc}
A & -P \\
0 & -A^{T}
\end{array}\right), \quad \bar{B}=\left(\begin{array}{c}
0 \\
C^{T}
\end{array}\right), \quad \bar{C}=(C
$$

yields

$$
\bar{Q} \bar{A} \bar{Q}^{-1}=\left(\begin{array}{cc}
A & 0 \\
0 & -A^{T}
\end{array}\right), \quad \bar{Q} \bar{B}=\left(\begin{array}{c}
Q C^{T} \\
C^{T}
\end{array}\right), \quad \bar{C} \bar{Q}^{-1}=\left(\begin{array}{ll}
C & -C Q
\end{array}\right) .
$$

Then $\left(A, Q C^{T}, C\right)$ is a minimal triple with $A$ asymptotically stable. When we define $Z(s)=C(I s-A)^{-1} Q C^{T}$, it follows that we have obtained a partial fraction expansion of $\Phi(s)$ since $\Phi(s)=Z(s)+Z^{T}(-s)$. 
Recapitulating we have obtained the following

\section{AlgorithM}

Data. $\Phi(s)$, the spectral density matrix of the process $y$.

Step 1. Construct a minimal realization $(\bar{A}, \bar{B}, \bar{C})$ of $\Phi(s)$. Find the unique nonsingular $J$ satisfying $\bar{A}^{T} J+J \bar{A}=0, \bar{B}^{T} J=\bar{C}$. Then also $J=-J^{T}$.

Step 2. Find a nonsingular $\Sigma$ satisfying $\bar{A}^{T} \Sigma+\Sigma \bar{A} \leqq 0, \bar{B}^{T} \Sigma=\bar{C}, \Sigma=J \Sigma^{-1} J$, $\Sigma=\Sigma^{T}$.

Step 3. Compute $\Sigma^{+}=\operatorname{ker}\left(I-J^{-1} \Sigma\right)$ and $\Sigma^{-}=\operatorname{ker}\left(I+J^{-1} \Sigma\right)$. Take a basis $\left(q_{1}, \cdots, q_{n}\right)$ for $\Sigma^{+}$and construct the basis $\left(p_{1}, \cdots, p_{n}\right)$ for $\Sigma^{-}$such that $q_{i}^{T} J p_{j}=\delta_{i j}, i, j=1, \cdots, n$.

In this basis we can write

$$
\bar{A}=\left(\begin{array}{cc}
F & -P \\
-R & -F^{T}
\end{array}\right), \quad \bar{B}=\left(\begin{array}{c}
0 \\
H^{T}
\end{array}\right), \quad \bar{C}=(H
$$

and we will have $P=P^{T} \geqq 0$ and $R=R^{T} \geqq 0$.

Step 4. Find the maximal symmetric nonnegative definite solution $K$ of

$$
F^{T} K+K F-K P K+R=0 .
$$

Step 5. Define $A:=F-P K, C:=H$, and $Q=Q^{T}>0$ as the unique solution of $Q A^{T}+A Q=-P$. Then $(A, Q, C)$ is a minimal realization of $y$.

Step 6. Let $P=B B^{T}$. Then

$$
\begin{aligned}
& d x=A x d t+B d w, \quad x(0)=x_{0}, \mathbf{E}\left\{x_{0} x_{0}^{T}\right\}=Q, \\
& \tilde{y}=C x
\end{aligned}
$$

is a white noise representation of $y$.

Remark 8. With respect to the actual calculation of $\Sigma$ in Step 2 we can be more specific. Recall that the signature triple of a symmetric matrix consists of the number of positive, negative, respectively zero eigenvalues. We make use of the following

LEMMA 8. Let $(\bar{A}, \bar{B}, \bar{C})$ be a minimal triple (not necessarily satisfying (1) and (2)). Denote the set of symmetric solutions of (3) and (4) by $S(\Sigma)$. Then every element of $S(\Sigma)$ is nonsingular and has the same signature triple. If $(\bar{A}, \bar{B}, \bar{C})$ also satisfies (1) and (2) then every element of $S(\Sigma)$ has $n$ negative eigenvalues and $n$ positive eigenvalues.

Proof. Let $\bar{A}^{T} \Sigma+\Sigma \bar{A}=-L^{T} L$. First suppose that $(\bar{A}, L)$ is observable. Now let $x \in \operatorname{Ker} \Sigma$. Then $x^{T} \bar{A}^{T} \Sigma x+x^{T} \Sigma \bar{A} x=-x^{T} L^{T} L x$ implies $L x=0$. Furthermore $\bar{A}^{T} \Sigma x+$ $\Sigma \bar{A} x=-L^{T} L x$ implies $\Sigma \bar{A} x=0$. Therefore $\operatorname{Ker} \Sigma$ is $\bar{A}$-invariant and contained in $\operatorname{Ker} L$. By observability of $(\bar{A}, L)$ it follows that $\operatorname{Ker} \Sigma=0$. If $(\bar{A}, L)$ is not observable we proceed as follows. By (3) and (4)

$$
(\bar{A}-\bar{B} \bar{C})^{T} \Sigma+\Sigma(\bar{A}-\bar{B} \bar{C})=-L^{T} L-2 \bar{C}^{T} \bar{C} .
$$

Then: $\{(\bar{A}, \bar{C})$ observable $\} \Leftrightarrow\left\{\left(\bar{A}, \bar{C}^{T} \bar{C}\right)\right.$ observable $\} \Leftrightarrow\left\{\left(\bar{A}-\bar{B} \bar{C}, 2 \bar{C}^{T} \bar{C}\right)\right.$ observable $\} \Rightarrow$ $\left\{\left(\bar{A}-\bar{B} \bar{C},-L^{T} L-2 \bar{C}^{T} \bar{C}\right)\right.$ observable $\}$. Therefore as above we can conclude that $\Sigma$ satisfying (15) is nonsingular.

Because $S(\Sigma)$ is convex and consists of nonsingular matrices every element of $S(\Sigma)$ has the same signature triple. By Lemma 4 it follows that there exists a $\Sigma \in S(\Sigma)$ satisfying (5). This $\Sigma$ has $n$ negative and $n$ positive eigenvalues as can be seen from (8).

Consider now the following algorithm-(see also [15]).

Let $\Sigma_{1}$ be a solution of (3) and (4). Then define

$$
\Sigma_{n+1}=\frac{1}{2}\left(\Sigma_{n}+J \Sigma_{n}^{-1} J\right), \quad n \geqq 1 \text {. }
$$


If $\Sigma \in S(\Sigma)$, then also $J \Sigma^{-1} J \in S(\Sigma)$. Therefore since every element of $S(\Sigma)$ is nonsingular and $S(\Sigma)$ is convex, $\Sigma_{n+1} \in S(\Sigma)$ for every $n \geqq 1$. From the compactness of $S(\Sigma)$ it follows that $\lim _{n \rightarrow \infty} \Sigma_{n} \in S(\Sigma)$. Denote $\Sigma_{\infty}:=\lim \Sigma_{n}$. Then $\Sigma_{\infty}$ satisfies $\Sigma_{\infty}=$ $\frac{1}{2}\left(\Sigma_{\infty}+J \Sigma_{\infty}^{-1} J\right)$, or equivalently, $\Sigma_{\infty}=J \Sigma_{\infty}^{-1} J$. Furthermore note that we can rewrite (16) as

$$
\Sigma_{n+1} J=\frac{1}{2}\left(\Sigma_{n} J+\left(\Sigma_{n} J\right)^{-1}\right) .
$$

Since $\lim \frac{1}{2}\left(\Sigma_{n} J+\left(\Sigma_{n} J\right)^{-1}\right)$ equals sign $\left(\Sigma_{n} J\right)$ (see e.g. [15]), $\Sigma_{\infty}$ may be written as (sign $\left.\left(\Sigma_{n} J\right)\right) J^{-1}$. Concluding, a solution $\Sigma$ satisfying (3), (4) and (5) may be computed as follows. Take any solution $\Sigma_{1}$ of (3) and (4) (this is a standard problem, see [5]). Compute $\Sigma_{\infty}:=\left(\operatorname{sign}\left(\Sigma_{1} J\right)\right) J^{-1}$. Then $\Sigma_{\infty}$ satisfies (3), (4) and (5).

Remark 9. The covariance $Q$ in Step 5, uniquely determined by $Q A^{T}+A Q=-P$, can also be computed directly from the Riccati equation of Step 4. In fact:

LEMMA. 9. Let

$$
\bar{A}=\left(\begin{array}{cc}
F & -P \\
-R & -F^{T}
\end{array}\right), \quad \bar{B}=\left(\begin{array}{c}
0 \\
H^{T}
\end{array}\right) \quad C=\left(\begin{array}{ll}
H & 0
\end{array}\right)
$$

be a minimal realization of a spectral density $\Phi(s)$, with $P \geqq 0, R \geqq 0$ (such a realization exists by Theorem 5). From Theorem 7 it follows that $(\bar{A}, \bar{B}, \bar{C})$ determines a minimal stochastic realization denoted by $(A, Q, C)$. Denote by $K^{+}$and $K^{-}$respectively the maximal and minimal symmetric solutions of the Riccati equation associated to $\bar{A}(11)$ :

$$
F^{T} K+K F+K P F-R=0 .
$$

Then $Q=\left(K^{+}-K^{-}\right)^{-1}$ (under the conditions of Theorem $7, K^{+}-K^{-}$is necessarily $>0$, cf. [13]).

Proof. After the basis transformation $\left({ }_{-K^{+}}^{I}{ }_{I}^{0}\right), \bar{A}$ is given by

$$
\left(\begin{array}{cc}
F^{+} & -P \\
0 & -\left(F^{+}\right)^{T}
\end{array}\right) \text { with } F^{+}=F-P K^{+}
$$

Let $\left(\begin{array}{c}-Q \\ I\end{array}\right) x$ be a vector in $\operatorname{Im}\left(\begin{array}{c}-Q \\ I\end{array}\right)$. Then, since $F^{+} Q+Q F^{+}=-P($ see $(13))$ :

$$
\left(\begin{array}{cc}
F^{+} & -P \\
0 & -\left(F^{+}\right)^{T}
\end{array}\right)\left(\begin{array}{c}
-Q \\
I
\end{array}\right) x=\left(\begin{array}{c}
-F^{+} Q-P \\
-\left(F^{+}\right)^{T}
\end{array}\right) x=-\left(\begin{array}{c}
-Q \\
I
\end{array}\right)\left(F^{+}\right)^{T} x .
$$

Therefore, in this new basis, $\operatorname{Im}\left(\begin{array}{c}-Q \\ I\end{array}\right)$ equals the positive eigenspace of $\bar{A}$ since $F^{+}$is asymptotically stable. Hence in the original basis the positive eigenspace of $\bar{A}$ is given by

$$
\left(\begin{array}{cc}
I & 0 \\
K^{+} & I
\end{array}\right) \operatorname{Im}\left(\begin{array}{c}
-Q \\
I
\end{array}\right)=\operatorname{Im}\left(\begin{array}{c}
-Q \\
-K^{+} Q+I
\end{array}\right)
$$

On the other hand we know that the positive eigenspace of $\bar{A}$ is also given by $\operatorname{Im}\left({ }_{K^{-}}^{I}\right)$. Therefore

$$
\operatorname{Im}\left(\begin{array}{c}
-Q \\
-K^{+} Q+I
\end{array}\right)=\operatorname{Im}\left(\begin{array}{c}
I \\
K^{-}
\end{array}\right)
$$

or $\left(K^{+}-K^{-}\right) Q=I$.

Remark 10. After having fixed a $\Sigma$ satisfying (3), (4) and (5), the stochastic realization $(A, Q, C)$ obtained in our algorithm is uniquely determined (up to basis transformations). The freedom in the choice of the covariance $Q$ as appearing in Theorem 2 (or alternatively the freedom in the choice of a spectral factorization of 
the spectral density, cf. [2] and Remark 7) is therefore equivalent to the freedom in the choice of a $\Sigma$ satisfying (3), (4) and (5).

Remark 11. Consider a stochastic vector process $y$ with autocorrelation function $R$ which admits a minimal white noise representation $d x=A x d t+B d w, \tilde{y}=C x$, as explained in Remark 1 . Then it is easy to see that the following equivalences hold: $\{y$ is ergodic $\} \Leftrightarrow\left\{\lim _{|t| \rightarrow \infty} R(t)=0\right\} \Leftrightarrow\{(A, B)$ is controllable $\} \Leftrightarrow\{\operatorname{Re} \sigma(A)<0\}$.

As we have seen in Theorem 7 , if we start from a rational spectral density $\Phi(s)$ we always arrive at a controllable, asymptotically stable white noise representation. Therefore a rational spectral density always corresponds to an ergodic process. On the other hand to give a stochastic realization of an autocorrelation function it is not necessary to assume that the process is ergodic. In some sense this is disadvantage of our approach. However the theory of $\S 3$ may be extended to cover the nonergodic case as well. It can be easily seen that the following is true. Let $(F, G, H)$ be a realization of $R$, i.e. $R^{+}(t)=H e^{F t} G$. Define the Hamiltonian system $(\bar{A}, \bar{B}, \bar{C})$ by

$$
\bar{A}=\left(\begin{array}{cc}
F & 0 \\
0 & -F^{T}
\end{array}\right), \quad \bar{B}=\left(\begin{array}{c}
G \\
H^{T}
\end{array}\right), \quad \bar{C}=\left(\begin{array}{ll}
H & -G^{T}
\end{array}\right) .
$$

Then:

$\{R$ is the autocorrelation function of an ergodic process $y\} \Leftrightarrow\{(\bar{A}, \bar{B}, \bar{C})$ is a minimal triple $\}$. If the process is ergodic (or $(\bar{A}, \bar{B}, \bar{C})$ is minimal) then it has spectral density $\Phi(s)=\bar{C}(I s-\bar{A})^{-1} \bar{B}$.

Finally we note that the spectral density of a nonergodic process is in a certain sense irrational. It is in fact of the form $\sum_{i=1}^{k} \pi\left(\delta\left(\omega-\omega_{i}\right)+\delta\left(\omega+\omega_{i}\right)\right)$, with $\left\{j \omega_{i}\right\}$ the spectrum of the generator of the nonergodic part (cf. [10]).

4. Conclusions. In this paper we have given a procedure for the stochastic realization of a spectral density matrix which starts by treating the spectral density as a (fictitious) transfer function. As such this transfer function has a symplectic structure and is passive. This structure is then exploited to arrive at a stochastic realization.

In comparison with the usual approach our method avoids having to factor the spectral density additively into its analytic and co-analytic part. However which of the two approaches would algorithmically be the most advantageous remains a matter of study.

\section{REFERENCES}

[1] R. Abraham And J. E. Marsden, Foundations of Mechanics, Benjamin/Cummings, New York, 1978.

[2] B. D. O. ANDERSON, The inverse problem of stationary covariance generation, J. Statist. Phys., 1 (1969), pp. 133-147.

[2] R. W. BROCKetT AND A. RAHIMI, Lie algebras and linear differential equations, in Ordinary Differential Equations, L. Weiss, ed., Academic Press, New York, 1972, pp. 379-386.

[4] P. FAurre, Réalisations Markoviennes de processus stationaires, Report IRIA, No. 13, 1973.

[5] P. Faurre, M. Clerget and F. Germain, Opérateurs rationnels positifs, Dunod, Paris, 1979.

[6] R. E. KALMAN, Linear stochastic filtering theory-Reappraisal and outlook, Proc. Brooklyn Polytechnic Symposium on System Theory, New York, 1965, pp. 197-205.

[7] V. KUCERA, A review of the matrix Riccati equation, Kybernetika, 9 (1973), pp. 42-61.

[8] A. LiNDQUiST AND G. PICCI, On the stochastic realization problem, this Journal, 17 (1979), pp. 365-390.

[9] K. R. MEYER, Hamiltonian systems with a discrete symmetry, J. Differential Equations, 41 (1981), pp. 228-238.

[10] A. PAPOulis, Probability, Random Variables, and Stochastic Processes, McGraw-Hill, New York, 1965. 
[11] G. RuckebuSH, Représentations markoviennes de processus gaussiens stationaires, Thesis, Univ de Paris VI, 1975.

[12] A. J. VAN DER SCHAFT, Time-reversible Hamiltonian systems, Systems \& Control Letters, 1 (1982), pp. 295-300.

[13] J. C. WILLEMS, Least squares stationary optimal control and the algebraic Riccati equation, IEEE Trans. Automat. Control, AC-16 (1971), pp. 621-634.

[14] - Realization of systems with internal passivity and symmetry constraints, J. Franklin Institute, 301 (1967), pp. 605-621.

[15] - Dissipative dynamical systems, Part II: Linear systems with quadratic supply rates, Arch. Rat. Mech. Anal., 45 (1972), pp. 352-393.

[16] A. M. Yaglom, An Introduction to the Theory of Stationary Random Functions, Dover, New York, 1973. 